\section{VACCINARE PER IL COVID-19 GLI ADOLESCENTI: LE RAGIONI E LE PAROLE DA DIRE}

Molto si è parlato in questi ultimi tempi dei diritti dell'uomo, ma è giunto il momento di parlare dei diritti sociali dei bambini e degli adolescenti, avrebbe detto Maria Montessori

Si potrebbe dire che ci siamo, che è arrivato il tempo vaccinale degli adolescenti, dai 12 anni in su. La raccomandazione dell'EMA (European Medicines Agency) arriva dopo quella della FDA (Food and Drug Administration) ed è stata trasmessa alla Commissione Europea. Spetta ora a ogni singolo Stato decidere se e quando usare questo vaccino negli adolescenti in futuro. L'AlFA (Agenzia Italiana del Farmaco) ne ha autorizzato I'uso in Italia e gli Organismi istituzionali (nelle parole del Ministro Speranza) dichiarano che "ora sarà possibile estendere la campagna di vaccinazione anche ai più giovani. È una novità importante, pensando anche alla riapertura delle scuole a settembre".

La prima Nazione ad avere iniziato la vaccinazione in questa fascia di età è stata il Canada, seguita dagli USA, e in Europa la Germania si è mossa tra le prime, così come I'Inghilterra.

Come pediatri, medici di Medicina generale e operatori sanitari dovremo informare ogni singolo adolescente (e i genitori) ed è quindi fondamentale condividere le ragioni di questa scelta. Volendo semplificare potremmo trovarci in sintonia con le parole del presidente dell'AIFA che dice nei titoli ripresi dagli organi di stampa: "I giovani sono un importante serbatoio di contagi. Importante vaccinarli". Aggiunge il presidente che: "ci sono già i vaccini e si completerà il ciclo per gli adolescenti dai 12 ai 15 anni che sono 8,5 milioni". Il presidente ha poi spiegato: "sebbene bambini e i giovani adolescenti si infettino meno e trasmettano meno il virus, è necessario vaccinare anche loro. Abbiamo imparato che le varianti hanno un maggiore indice di contagiosità e anche i bambini si infettano e possono essere una via di trasmissione, quindi possono essere un importante serbatoio. Per questo la vaccinazione degli adolescenti assume un ruolo strategico fondamentale per contenere i contagi: anche i ragazzi e le ragazze di 12-15 anni potranno prenotarsi per essere vaccinati".

Con riferimento alla nuova fase del piano, la Struttura Commissariale per l'Emergenza aggiunge: "le Regioni e le Province autonome dovranno garantire, prima dell'inizio delI'anno scolastico, la massima copertura possibile di tutta la popolazione studentesca, nelle fasce di età per le quali la vaccinazione sarà possibile, dunque dai 12 anni in poi".

Ho riflettuto molto su queste motivazioni e penso che il messaggio che noi potremmo dare dovrebbe trovare, per una volta, delle parole diverse, rivolte direttamente se possibile ai ragazzi/e da vaccinare e ai loro genitori. Non è facile trovarle ma dev'esserci una chiarezza informativa al meglio delle conoscenze disponibili. In una scelta come questa c'è sempre un'informazione che dev'essere in primo luogo individuale e che risponde a semplici domande che potrebbero essere le seguenti.

\section{Ma questa infezione se la dovessi prendere mi può fare stare male?}

La risposta è no, nella strangrande maggioranza dei casi. Come sai è una infezione a volte molto grave nelle persone di una certa età (i tuoi nonni) e in quelle che hanno malattie, ma nei giovani questo per fortuna non accade, anche se ci sono stati casi in cui l'infezione è stata un po' più importante anche in ragazzi della tua età, ma raramente.

\section{E allora perché dovrei farla?}

Dovresti farlo per quello che è successo e che potrà succedere, e per tutti i sacrifici che hai dovuto accettare (no scuola, no sport, no feste, pochi rapporti con amici). La vaccinazione potrà dare l'opportunità, a te e i tuoi amici, di tornare a una vita piena, non fatta solo di privazioni, e sarà un modo per ribadire in modo particolare i tuoi personali diritti e non solo quelli degli altri che potrebbero contagiarsi. Perché ci si potrebbe ammalare, perché tu ti sei ammalato, magari non sapendolo. Dunque sarà una scelta fatta per se stessi e non (solo) per gli altri. Sapendo anche che, se in una classe $o$ in un gruppo di amici si sceglierà di vaccinarsi, sarà come decidere di fare qualcosa di importante insieme, anche per garantire una buona scuola (che è anche e soprattutto relazione con gli altri), una normale vita sociale, insomma una vita che tu immagini e a cui ti stai disabituando, e altro ancora. Ti chiedo di dirmi tu quello che ora ti manca e che potresti ritrovare con la vaccinazione. Posso immaginarlo, ma avrei piacere di sentirlo da te.

\section{Potrei essere anche convinto, ma non so se questa vacci- nazione mi possa fare stare male.}

Nel corso degli anni ogni bambino e poi adolescente ha fatto tante vaccinazioni e senza problemi. Tu non lo ricordi bene, ma è così. Chiedilo ai tuoi genitori. Queste vaccinazioni sono state importanti per i bambini e i futuri adulti perché hanno evitato infezioni che un tempo erano pericolose per se stessi (e anche per gli altri) anche di più del coronavirus. E quello che stai vedendo e sentendo con i milioni di casi di adulti che si stanno vaccinando nel mondo, ti deve rendere fiducioso che le cose andranno bene. Il vaccino che devi fare è stato studiato: al momento ragazzi della

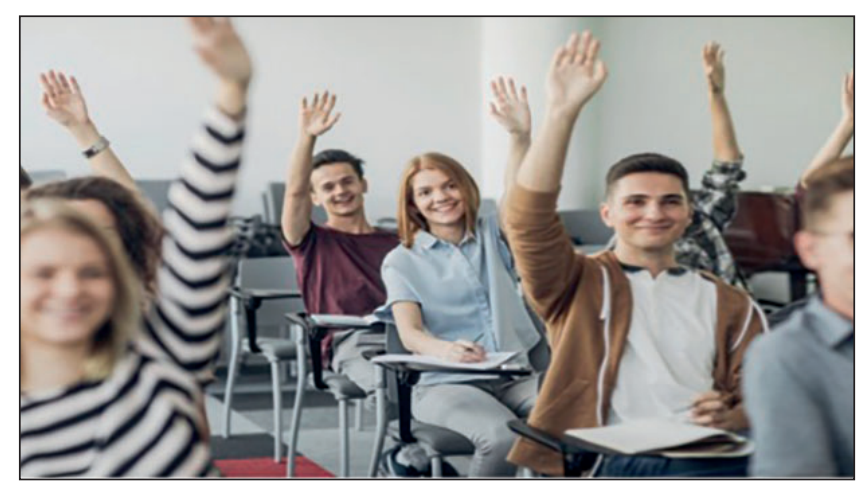


tua età che sono stati vaccinati non hanno avuto l'infezione da coronavirus nella totalità dei casi. Poi, come in tutte le cose, al pari dei rischi che posso esserci prendendo una medicina o andando in motorino, per quanto ci sia la massima accortezza e attenzione, può esserci qualche evento non previsto, raro, rarissimo. Quello che più comunemente potrà accadere è di avere un po' di dolore nel punto dell'iniezione, magari un po' di febbre, stanchezza o dolore alle ossa che per un giorno non ti farà andare a scuola, ma questa volta non dovrai fare la didattica a distanza (!), che sarà un ricordo di quelli che ti saranno stati utili per capire quanto sia importante "la presenza", quella vissuta, di ogni singola esperienza di vita.

\section{Ci penso, dovrò decidere con i miei genitori; c'è qualche altra cosa che dovrei sapere per fare la scelta migliore?}

Le dosi del vaccino saranno due, come per gli adulti. Farai la seconda dose dopo 21 giorni dalla prima.

Vorrei dirti un'ultima cosa, e te la dico più come padre o come persona che ha conosciuto tanti ragazzi/e come te: in questo lungo anno in tanti abbiamo pensato (e non siamo stati capaci di dirlo con le parole giuste), che voi ragazzi (e i bambini) siete la cosa più importante per noi, siete costantemente nei nostri pensieri. Il tuo futuro, e il vostro futu- ro, più di quello di altri è quello che ci interessa di più. Sono sicuro che il vaccino che potrai fare sarà una parte piccola, ma magari importante, per la tua serenità e felicità.

Ecco, parlerei così a un adolescente e ciascuno di noi troverà delle parole migliori e magari più convincenti; ma non gli direi che deve vaccinarsi per proteggere (anche questa volta) qualcuno. Semplicemente sarà importante che torni alla sua vita scolastica di tutti i giorni, a incontrare gli amici, magari senza la mascherina che ha nascosto il sorriso e anche la rabbia verso il mondo. Continuare a vivere come abbiamo vissuto per più di un anno è la vera "variante" della nostra esistenza.

$C^{\prime}$ è una speranza finale di questo dialogo vissuto e non immaginario con un adolescente nella scelta consapevole vaccinale: che anche i mezzi di comunicazione trovino il modo di dare voce ai ragazzi. Anche perché, come abbiamo spesso detto sulle pagine di Medico e Bambino, sono in grado di insegnarci qualcosa sul vero significato pubblico e scientifico della conoscenza, che ha nel dubbio, anche nel dubbio, le vere ragioni per un impegno sia personale che collettivo.

Federico Marchetti

\section{SOFFERMARSI}

Soffermarsi per una riflessione su cosa questa crisi planetaria dovuta alla pandemia ci ha insegnato è un dovere verso noi stessi e una preziosa opportunità di sintesi da condividere. Tra i tanti pensieri e le tante problematiche messe a fuoco riflettendo e chiacchierando in famiglia e in rete con Colleghi e amici, ho faticato a scegliere gli argomenti più opportuni per questa pubblicazione che, come un fiume carsico, è destinata a scomparire da subito negli scaffali delle nostre biblioteche, per poi riapparire nel tempo enne volte, anche solo per una lettura fugace.

Ho scelto due argomenti: uno negativo e uno positivo, per ragioni di equilibrio e impossibilità a definire quello più rilevante.

Il primo, negativo, è rappresentato dai limiti attuali del metodo scientifico. In questa prima vera prova da carico planetaria, in era digitale, la Scienza non ha saputo muoversi in maniera sufficientemente efficace, efficiente, coordinata e trasparente così da risultare utile per i cittadini del mondo. Nella pandemia la Medicina si è pubblicamente snaturata presentandosi come Scienza medica più che come Arte medica, in grado di parlare per tutte le altre scienze. II metodo utilizzato però ha dimostrato tutti i propri limiti. Limiti soprattutto di obsolescenza novecentesca da cui l'incapacità di accedere, gestire, interpretare e trasferire una quantità enorme di informazione generata da diverse componenti in ragione di un vero bisogno planetario. II Sapere, sotto stress, ha fornito troppo spesso risposte lente, talora solo di buon senso, non sempre corrette e quasi mai comunicate in maniera fruibile e non retorica. All'immenso Sapere della Scienza, che prevalentemente ha parlato per bocca dei medici, è mancata l'attenzione agli altri Saperi (economico, sociologico, filosofico, teologico) in grado, con lei e più di lei, di dare un senso all'apparentemente insensato. Declinata secondo un metodo rigido e superato la Scienza spesso ha parlato per se stessa perdendo consistenza e utilità per la vita dell'vomo che la ascoltava con l'attenzione di mai.

Il secondo, positivo, è rappresentato dall'opportunità che I'irruenza virale dell'infezione da SARS-CoV-2 ci ha dato di riproporre il tema della finitudine dell'vomo.

Nell'era della comunicazione e della tecnologia, il delirio di un paradigma di Medicina post-genomica di precisione, da cui ci si attendono sempre e comunque risposte efficaci per annullare l'effetto delle malattie e allontanare sempre più lo spettro della morte fino a scotomizzarlo, ha lasciato il posto all'ineluttabile e all'indicibile finitudine spesso gravata da un'inaccettabile solitudine. Occasione unica e importante per ritrovare il valore dell'accompagnamento, degli affetti e anche di una coraggiosa Medicina della persona capace di continuo contenimento e insostituibili carezze. Sapremo mantenere questo senso della nostra finitudine? Se sì, dovremo annoverare anche questo evento storico solo come un ulteriore passo del destino dell'vomo sul pianeta Terra.

Andrea Pession

Clinica Pediatrica

IRCCS Azienda Ospedaliero-Universitaria di Bologna 